\title{
Objective PET Lesion Segmentation Using a Spherical Mean Shift Algorithm
}

\author{
Thomas B. Sebastian ${ }^{1}$, Ravindra M. Manjeshwar ${ }^{1}$, Timothy J. Akhurst ${ }^{2}$, \\ and James V. Miller ${ }^{1}$ \\ ${ }^{1}$ GE Research, Niskayuna, NY \\ ${ }^{2}$ Department of Nuclear Medicine, MSK Cancer Center, New York, NY
}

\begin{abstract}
PET imagery is a valuable oncology tool for characterizing lesions and assessing lesion response to therapy. These assessments require accurate delineation of the lesion. This is a challenging task for clinicians due to small tumor sizes, blurred boundaries from the large point-spread-function and respiratory motion, inhomogeneous uptake, and nearby high uptake regions. These aspects have led to great variability in lesion assessment amongst clinicians. In this paper, we describe a segmentation algorithm for PET lesions which yields objective segmentations without operator variability. The technique is based on the mean shift algorithm, applied in a spherical coordinate frame to yield a directional assessment of foreground and background and a varying background model. We analyze the algorithm using clinically relevant hybrid digital phantoms and illustrate its effectiveness relative to other techniques.
\end{abstract}

\section{Introduction}

FDG PET and FDG PET+CT allow clinicians to make inferences on the nature of lesions from local measures of metabolic rate. Clinicians can infer lesion characterization (inflammation verses cancer), establish lesion boundaries for radiation therapy, and estimate response to therapy from the FDG PET local measures of metabolic rate. These tasks require clinicians to estimate the boundaries of lesions in three dimensions and summarize the metabolic activity over these three dimensional regions. These are challenging tasks due to the inhomogeneity of the metabolic rate across the lesion, the inhomogeneity of the metabolic rate across the background, and the potential for high metabolic rates in nearby anatomy. Furthermore, lesions can be small relative to the point-spread-function of the imaging device and their boundaries may be blurred by respiratory motion during long image acquisitions. Figure 1 illustrates some of the challenges in estimating boundaries and activity.

These challenges have led clinicians to use simple criteria to evaluate lesions. For instance, simple window-level and thresholding techniques are often used for segmenting lesions in FDG PET and the maximum uptake over a region is used to summarize the activity over the lesion. These simple approaches are suboptimal for quantifying activity and lead to a large degree of variability 

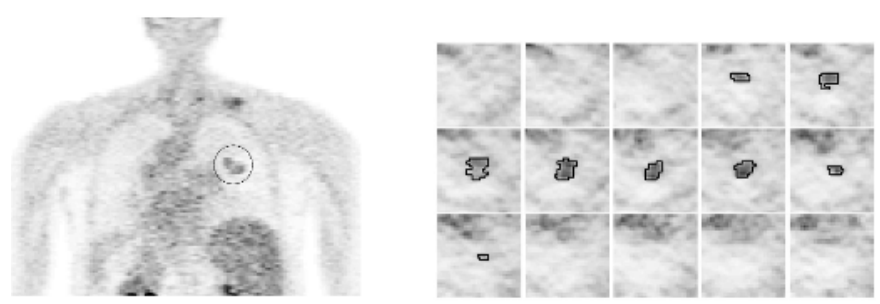

Fig. 1. Left: Example lesion imaged with PET. Right: Cropped axial slices of the same lesion with segmentation using proposed approach overlaid.

amongst clinicians. Hence, the goal of this paper is to develop an objective FDG PET lesion segmentation algorithm that requires minimal user interaction.

Several algorithms have been proposed for medical image segmentation. However, we found many traditional segmentation algorithms failed to properly segment PET lesions. Gradient based techniques failed because of difficulty in estimating reliable gradients on small or diffused lesions. Region based techniques failed because the interior of a lesion is considerably inhomogeneous. Classification based techniques [12] applied at the pixel level failed because of the inhomogeneity of the interior of the lesion and the inhomogeneity of the background. Classification methods that included neighborhood constraints [34] had some success but failed when the background varied around the lesion boundary.

In this paper, we present a lesion segmentation technique that provides clinicians with an objective estimation of lesion boundaries from which they can make inferences on lesion characterization, plan radiation therapy, and estimate response to therapy. The main challenge in segmenting PET lesions is the inhomogeneous nature of the lesion and the background. We apply the mean shift algorithm in a spherical coordinate frame which facilitates varying background models around the lesion boundary. The piecewise constant approximation produced by the spherical mean shift is used to estimate a directionally varying background model, which is then used to classify the background and foreground of the lesion. The spherical mean shift (SMS) algorithm is particularly effective in segmenting lesions that are adjacent to normal tissue with high uptake like the liver or mediastinum, and inhomogeneous lesions.

Validation of segmentation algorithms is challenging because of the difficulty in obtaining reproducible ground-truth. To address this problem for PET lesions, we use hybrid digital phantoms [5]. Models of lobulated lesions are projected into sinogram space, combined with sinograms from real subjects, and reconstructed using a PET simulator that accurately models the point-spread-function and noise properties of a real PET system. The hybrid digital phantoms provide images which are indistinguishable from real lesions [5] while allowing for precise control over the ground truth. The SMS algorithm is shown to outperform two other algorithms: neighborhood expectation maximization (NEM) and spatiallyweighted fuzzy clustering (SFCM). 


\section{Mean Shift}

Fukunaga and Hostetler introduced the mean shift algorithm 67] to estimate the gradient of a probability density function given a set of samples from the distribution. Using a hill climbing algorithm, the gradient estimate can be used to identify the modes of the underlying distribution. The mean shift algorithm has been used for clustering [6], segmentation [8], and tracking 9].

Let $\left\{\boldsymbol{x}_{\boldsymbol{i}}\right\}$ denote the set of $n d$-dimensional samples from the distribution and let $\boldsymbol{f}_{\boldsymbol{i}}^{\boldsymbol{j}}$ denote each sample at the $j^{\text {th }}$ mean shift iteration where $\boldsymbol{f}_{\boldsymbol{i}}^{\mathbf{0}}=\boldsymbol{x}_{\boldsymbol{i}}$. The mean shift algorithm is

$$
f_{i}^{j+1}=f_{i}^{j}+M\left(f_{i}^{j}\right)
$$

where $M\left(f_{i}^{j}\right)$ is the mean shift vector

$$
\boldsymbol{M}\left(\boldsymbol{f}_{\boldsymbol{i}}^{\boldsymbol{j}}\right)=\frac{\sum_{k=1}^{n} \boldsymbol{f}_{\boldsymbol{k}}^{\boldsymbol{j}} w\left(\left\|\frac{\boldsymbol{f}_{\boldsymbol{k}}^{\boldsymbol{j}}-\boldsymbol{f}_{\boldsymbol{i}}^{\mathbf{0}}}{h}\right\|^{2}\right)}{\sum_{k=1}^{n} w\left(\left\|\frac{\boldsymbol{f}_{\boldsymbol{k}}^{\boldsymbol{j}}-\boldsymbol{f}_{\boldsymbol{i}}^{\mathbf{0}}}{h}\right\|^{2}\right)}-\boldsymbol{f}_{\boldsymbol{i}}^{\mathbf{0}}
$$

and $w$ is a non-negative, non-increasing weight function and $h$ is a bandwidth parameter.

To apply mean shift to image segmentation [8, each pixel is treated as a $d$ dimensional sample of its position and intensity, $\boldsymbol{f}_{\boldsymbol{i}}^{\mathbf{0}}=[x, y, z, I(x, y, z)]^{\prime}$. The mean shift algorithm iteratively moves the samples, converging on the modes of the joint spatial+intensity domain. Pixels that converge to the same mode are labeled as being from the same segment.

\section{Spherical Mean Shift}

The straightforward application of the mean shift to PET lesions fails to properly segment small lesions and lesions with diffuse boundaries. The algorithm merges these lesions into the background. For larger lesions, the mean shift algorithm produces a segmentation that underestimates the volume of convex lesions.

To address these issues, we apply the mean shift algorithm to a lesion that has been resampled into spherical coordinates. First, we define a small region of interest (ROI) around the lesion in Cartesian space, centered on the lesion and comfortably sized at approximately $3 \times$ the size of the lesion. The imagery within the ROI is resampled into spherical coordinates: radial distance to the center, $r$, azimuthal angle in the $x y$-plane, $\theta$, and polar angle from the $z$-axis, $\phi$

$$
x=r \cos \theta \sin \phi, \quad y=r \sin \theta \sin \phi, \quad z=r \cos \phi .
$$

where $r=[0, \infty], \theta=[0,2 \pi]$ and $\phi=[-\pi, \pi]$. The resampling results in an image indexed by $r, \theta$, and $\phi$. We refer to this resampling process as lesion unwrapping.

We could apply the mean shift algorithm along each radial line independently, obtaining a piecewise constant approximation of the image intensity along each 
radial direction, as in [10] in the study of vessel cross-sections. However, processing each radial line independently results in piecewise constant approximations that are inconsistent across radial lines, as azimuthal and polar spatial information is not utilized. Therefore, we apply the mean shift algorithm over a neighborhood of radial lines in the unwrapped lesion. The samples in the mean shift are the voxel's spatial coordinates and intensity, $\boldsymbol{f}_{\boldsymbol{j}}^{\mathbf{0}}=[r, \theta, \phi, I(r, \theta, \phi)]^{\prime}$. The bandwidths for the mean shift procedure are chosen such that $h_{\theta} \approx h_{\phi} \ll h_{r}$. The intensity (range) bandwidth is estimated as a function of the intensity value at the center of the ROI. To compute the mean shift vector, we use a kernel of size $21 \times 3 \times 3(r, \theta, \phi)$, which corresponds to a cone in the spherical coordinate frame.

The spherical mean shift algorithm iteratively moves the samples, converging on the modes of the joint (spherical) spatial+intensity domain. Voxels that converge to the same mode are labeled as being from the same segment.

\section{Directional Statistical Background Model}

The spherical mean shift algorithm of Section 3 results in a piecewise constant approximation of the signal intensity. This initial segmentation is used to estimate a directionally varying statistical model of the background. In most cases, a global model of the background will not suffice because of the significant variation in the background on different sides of the lesion. For example, a lesion that is adjacent to high-uptake regions such as heart or mediastinum may have higher background levels to one side. To address this variation, we estimate a different statistical model for the background in each radial direction.

The background model for each radial line is once again based on a neighborhood of radial lines. We identify all the voxels in the bundle of the neighborhood that are associated with the segment with the lowest intensity in the initial spherical mean shift segmentation. A robust estimate of the minimum can be obtained by not considering small regions in this step. The statistical model of the background in the given radial direction is estimated from the original PET image intensity values of these candidate voxels. Currently, we use a simple statistical model composed of the sample mean $\mu_{b}$ and the standard deviation $\sigma_{b}$. All voxels within $\mu_{b} \pm \sigma_{b}$ are assigned to the background and the remaining voxels to the lesion. By repeating this process on all radial lines, we segment the lesion in spherical coordinates. Finally, we resample the segmentation back into Cartesian coordinates using nearest neighbor interpolation.

\section{Lesion Database}

Phantom acquisitions are routinely used for performance evaluation of segmentation algorithms because they provide reproducible, ground-truth information. However, these phantoms typically represent geometrically-simple shapes and uniform activity distributions superimposed on homogeneous backgrounds. This scenario differs dramatically from the complex shapes of lesions and the inhomogeneous lesion and background activity distributions encountered in clinical 


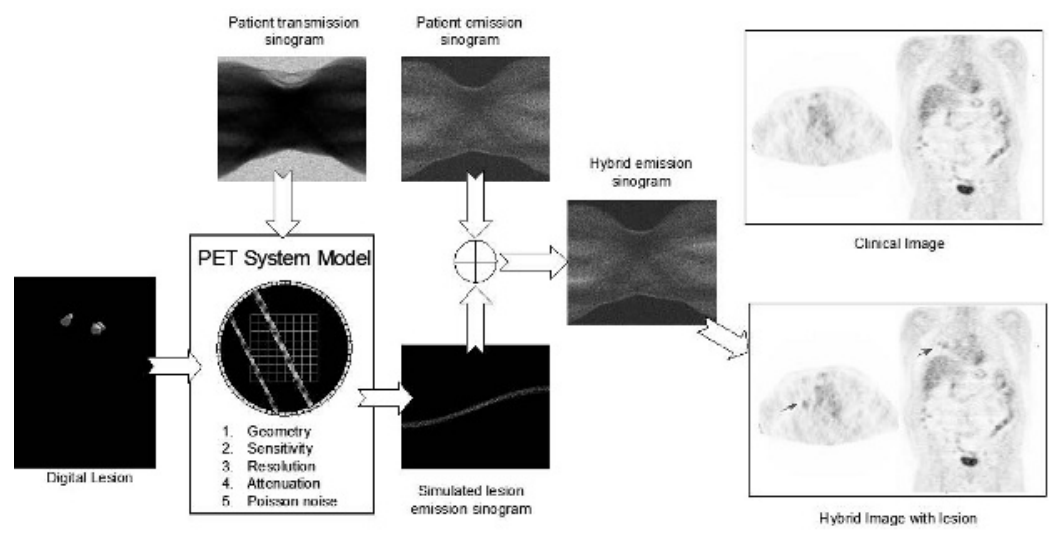

Fig. 2. Schematic diagram illustrating how hybrid phantoms are generated

PET images. Therefore, phantom images may not provide a clinically relevant and/or adequate challenge in evaluating segmentation algorithms.

To address this concern, we use hybrid phantoms, consisting of digital lesions embedded seamlessly into actual FDG PET patient data sets [5], Figure 2, Digital lesions of random shapes, sizes and non-uniform voxel activity distributions were constructed from combinations of ellipsoidal primitives. An analytical simulation of the GE Advance $\mathrm{NXi}^{\mathrm{TM}}$ PET scanner operating in 2D acquisition mode was used to generate emission sinograms of the digital lesions. The PET acquisition simulation modeled the attenuation of activity from the lesions due to patient tissue as well as the physical properties of the PET scanner (spatial resolution, energy resolution and Poisson statistics). The lesion emission sinograms were attenuated using patient transmission data and added to patient FDG emission sinograms that were corrected for decay, randoms and scatter, but not attenuation. The combined emission sinograms were reconstructed to seamlessly embed the digital lesion into clinical patient data. It must be emphasized that the digital lesions were embedded into patient data in the sinogram domain and not in the image domain. The hybrid images generated by this technique have been determined to be indistinguishable from clinical images by an expert nuclear medicine physician (with experience in reading more than 5000 scans) [5].

The reconstructed images contain ground truth lesions with differing patterns of FDG uptake, a variety of shapes and sizes, and clinically relevant background textures and uptake from adjacent anatomies. These hybrid phantoms provide a realistic challenge to a segmentation algorithm, yet contain information of the ground truth, which cannot be obtained from actual patient tumors.

Thirty-five clinical reference patient datasets acquired on the GE Advance $\mathrm{NXi}^{\mathrm{TM}}$ scanner, were selected for their low tumor burden in the lungs. Two hundred and eighty digital lesions with volumes of 5-40 $\mathrm{ml}$ and maximum sourceto-background ratios ranging from $3: 1$ to $6: 1$ were generated. The digital lesions were embedded inside the lung region of the reference patient datasets with some lesions positioned in isolation, others close to the mediastinum and the remaining 
close to the lung wall. All images were reconstructed with the iterative OSEM $(28 / 2)$ algorithm on a $128 \times 128$ grid with a pixel size of $4.29 \mathrm{~mm}$ and slice thickness of $4.25 \mathrm{~mm}$.

\section{Results}

We illustrate the performance of the spherical mean shift algorithm in Figures 3 4. Figure 3 shows three cases with the challenges mentioned in Section 1 Figure 4 shows a lesion segmented with the spherical mean shift (SMS) algorithm and compares it against the results from a spatially-weighted fuzzy clustering method (SFCM) and a neighborhood expectation maximization method (NEM).

(a)
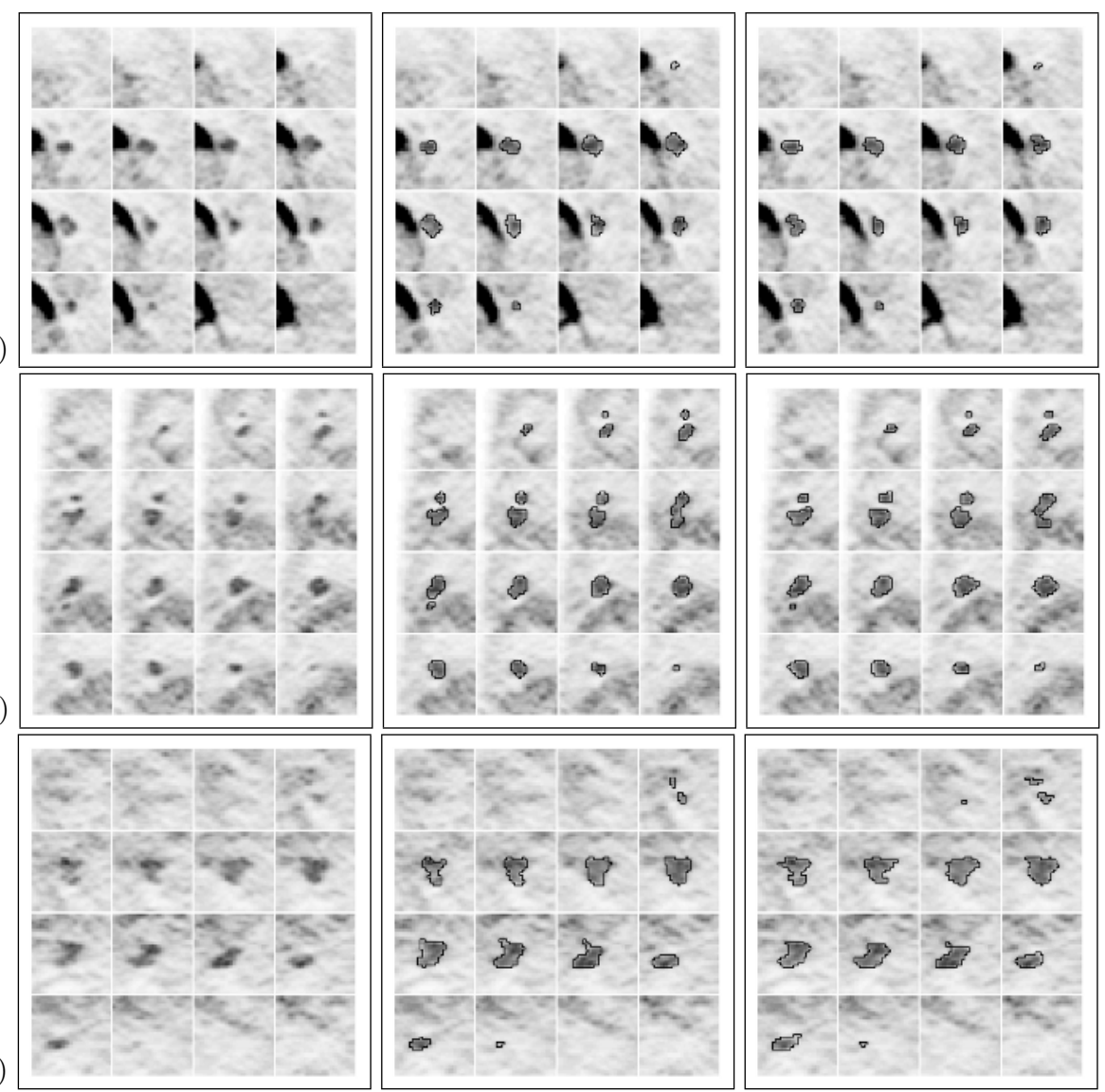

Fig. 3. SMS segmentation results. Columns show the original image, ground truth, and segmentation results. SMS produces a viable segmentation when (a) the lesion is adjacent to another region of high uptake, (b) the lesion has a complex topology, and (c) the lesion is inhomogeneous and has poor contrast. 


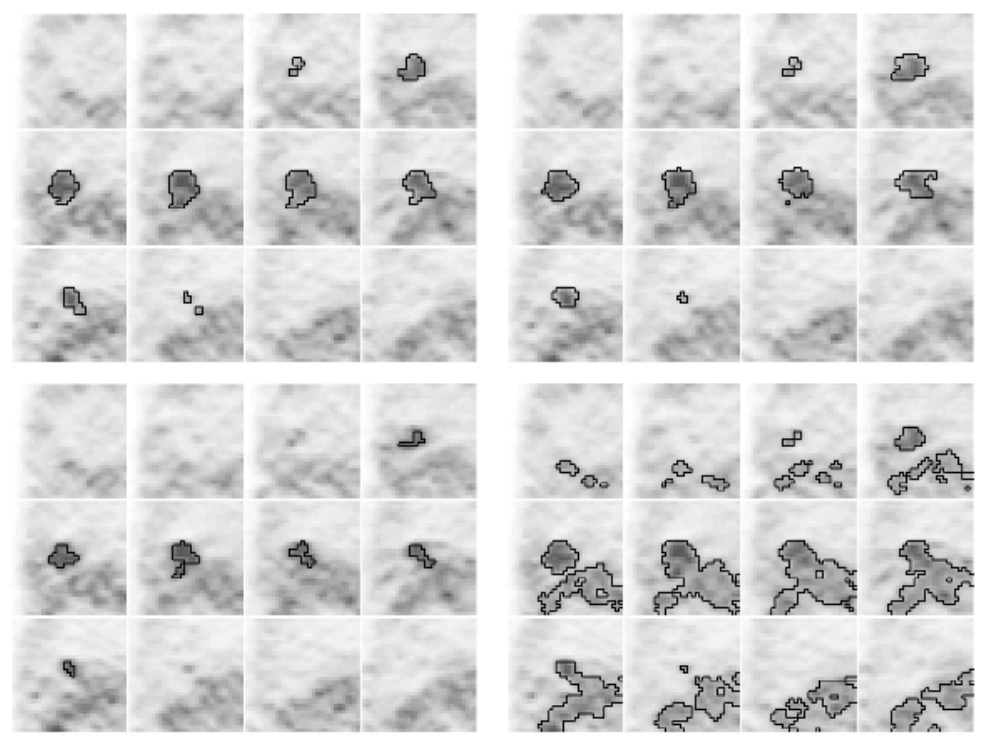

Fig. 4. Segmentation comparison. Clockwise from top left: True lesion, SMS, SFCM, NEM.

Table 1. Algorithm comparison on 280 hybrid digital phantoms of various sizes

\begin{tabular}{|c|c|c|c|c|c|c|}
\hline & \multicolumn{2}{|c|}{ SFCM } & \multicolumn{2}{c|}{ NEM } & \multicolumn{2}{c|}{ SMS } \\
\hline & \#Fail & DSC & \#Fail & DSC & \#Fail & DSC \\
\hline $5 \mathrm{cc}$ & 25 & $0.336 \pm 0.357$ & 11 & $0.573 \pm 0.312$ & 4 & $0.731 \pm 0.171$ \\
\hline $10 \mathrm{cc}$ & 20 & $0.408 \pm 0.357$ & 9 & $0.702 \pm 0.242$ & 3 & $0.805 \pm 0.096$ \\
\hline $25 \mathrm{cc}$ & 15 & $0.379 \pm 0.336$ & 11 & $0.642 \pm 0.309$ & 0 & $0.812 \pm 0.094$ \\
\hline $40 \mathrm{cc}$ & 13 & $0.596 \pm 0.322$ & 8 & $0.760 \pm 0.222$ & 0 & $0.858 \pm 0.065$ \\
\hline
\end{tabular}

To further quantify the performance of the spherical mean shift, we apply the algorithm to the aforementioned hybrid phantom lesions. Table 1 summarizes the results of SMS and provides a comparison to SFCM and NEM. If an algorithm generated a gross oversegmentation - mislabeling more than half of the foreground as background, or generated a gross undersegmentation - mislabeling more background as foreground as there is foreground in the ground truth, then the algorithm was said to have failed on that lesion. Dice Similarity Coefficients (DSC) 11

$$
D S C(A, B)=\frac{2|A \cap B|}{|A|+|B|}
$$

were calculated and means and standard deviations of the DSC scores are reported for each algorithm and each nodule size. From the table, it is apparent that the spherical mean shift is more robust since it succeeds in producing a segmentation $97.5 \%$ of the time (compared to $\mathrm{SFCM}=74 \%$ and $\mathrm{NEM}=86 \%$ ). The 
spherical mean shift is more accurate as it achieves higher DSC values for every lesion size tested. Finally, the spherical mean shift is more precise as it achieves lower standard deviations in DSC values.

\section{Conclusions}

We present an objective segmentation algorithm that can robustly, accurately, and precisely segment small lesions is PET imagery. We use the mean shift algorithm, applied in a spherical coordinate frame, to produce a directionally varying background model. This background model is used to classify the foreground and background along each radial direction. We analyze the algorithm using 280 clinically relevant hybrid digital phantoms and qualitatively and quantitatively compare the spherical mean shift algorithm to two other algorithms.

\section{References}

1. N. Otsu. A threshold selection method from gray level histograms. T-SMC, 1:6266, 1979 .

2. A. Dempster, N. Laird, and D. Rubin. Maximum likelihood from incomplete data via the EM algorithm. Journal of Royal Statistical Society, Series B, 39:1-38, 1977.

3. C. Ambroise, M. Dang, and G. Govaert. Clustering of spatial data by the EM algorithm. In Geostatistics for Environmental Applications, volume 9 of Quantitative Geology and Geostatistics, pages 493-504. Kluwer Academic Publishers, 1997.

4. Y. Tolias and S. Panas. Image segmentation by a fuzzy clustering algorithm using adaptive spatially constrained membership functions. T-SMC, 28(3):359-369, 1998.

5. R.M. Manjeshwar, T.J. Akhurst, B.B. Beattie, H.E. Cline, D.E. Gonzalez Trotter, and J.L. Humm. Realistic hybrid digital phantoms for evaluation of image analysis tools in positron emission tomography. In SNM, 2002.

6. K. Fukunaga and L.D. Hostetler. The estimation of the gradient of a density function, with applications in pattern recognition. T-IT, 21(1):32-40, 1975.

7. Y. Cheng. Mean shift, mode seeking and clustering. T-PAMI, 17(8):790-799, 1995.

8. D. Comaniciu and P. Meer. Mean shift: A robust approach toward featue space analysis. T-PAMI, 24(5):603-619, 2002.

9. D. Comaniciu, V. Ramesh, and P. Meer. Real-time tracking of non-rigid objects using mean shift. In CVPR 2000, volume 2, pages 142-149, 2000.

10. H. Tek, D. Comaniciu, and J. Williams. Vessel detection by mean-shift based ray propagation. In $M M B I A$, pages 228-235, 2001.

11. L.R. Dice. Measures of the amount of ecologic association between species. Ecology, 26:297-302, 1945 . 